УДК 681.7 .068

\author{
В.П. Первадчук ${ }^{1}$, Д.Б. Владимирова ${ }^{1}$, \\ Д.Н. Дектярев ${ }^{1}$, А.А. Пестерев ${ }^{2}$ \\ ${ }^{1}$ Пермский национальный исследовательский \\ политехнический университет, Пермь, Россия \\ ${ }^{2} \mathrm{OAO}$ «Пермская научно-производственная \\ приборостроительная компания», Пермь, Россия \\ ПРОЕКТИРОВАНИЕ ОПТИМАЛЬНОГО \\ СТАБИЛИЗИРУЮЩЕГО РЕГУЛЯТОРА \\ В MCVD-ПPOЦЕCCE
}

В настоящее время активно развивается теория оптимального управления систем с распределенными параметрами, в том числе систем с подвижным источником воздействия. Температурное поле в процессе MCVD характеризуется пространственной протяженностью, и его следует рассматривать как объект с распределенными параметрами. В данной работе рассматриваются вопросы проектирования оптимального стабилизирующего регулятора для управления температурным полем в процессе MCVD.

Ключевые слова: оптимальное управление, оптимальный стабилизирующий регулятор, подвижный тепловой источник, MCVD-процесс.

\author{
V.P. Pervadchuk ${ }^{1}$, D.B. Vladimirova ${ }^{1}$, \\ D.N. Dektiarev ${ }^{1}$, A.A. Pesterev ${ }^{2}$ \\ ${ }^{1}$ Perm National Research Polytechnic University, Perm, Russian Federation \\ ${ }^{2}$ Perm Scientific-Industrial Instrument Making Company, \\ Perm, Russian Federation
}

\title{
DESIGNING OF OPTIMAL STABILIZING REGULATOR IN THE MCVD PROCESS
}

Now, the theory of optimal control of systems with distributed parameters is actively developing, including systems with a moving heat source. The temperature field in the MCVD process is characterized by spatial extent and should be considered as an object with distributed parameters. In this paper, we will consider the design process of an optimal stabilizing regulator for controlling the temperature field in the MCVD process.

Keywords: optimal control, optimal stabilizing regulator, moving heat source, MCVD process. 


\section{Введение}

Технология получения кварцевых заготовок методом MCVD (Modified Chemical Vapor Deposition) была открыта еще в конце 70-х гг. прошлого века. Универсальность MCVD-технологии заключается в возможности изготовления кварцевых заготовок требуемого качества практически для всех видов оптических волокон.

На рис. 1 схематически показан процесс MCVD. В опорную кварцевую трубку подают пары четыреххлористого кремния $\left(\mathrm{SiCl}_{4}\right)$ и галогенидов легирующих компонентов $\left(\mathrm{GeCl}_{4}, \mathrm{BCl}_{3}, \mathrm{POCl}_{3}\right.$ и т.д.), а также очищенный и осушенный кислород.
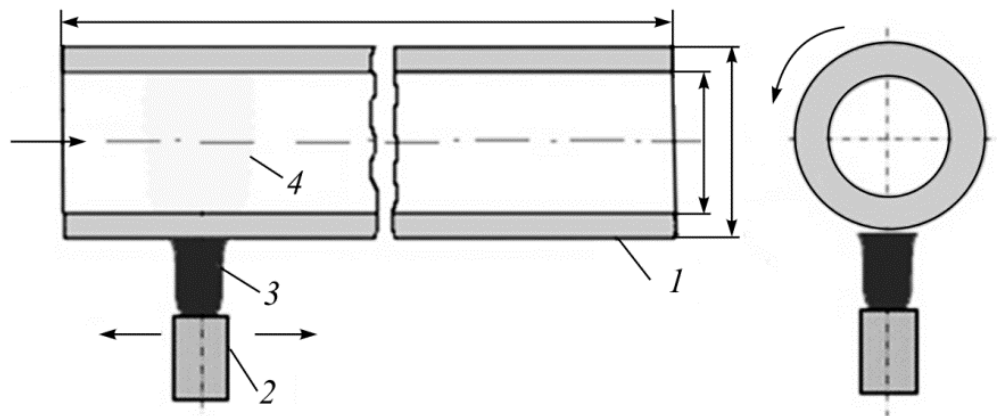

Рис. 1. Схематическое изображение процесса MCVD: 1 - кварцевая труба;

2 - движущаяся горелка (стрелки показывают направление движения); 3 - пламя горелки; 4 - зона реакции

В зоне нагрева трубки кислородно-водородной горелкой, перемещающейся вдоль трубки с заданной скоростью (100-120 мм/мин), происходит окисление галогенидов с образованием оксидов кремния и легирующих компонентов. Далее эти оксиды осаждаются за счет термофореза на внутреннюю поверхность опорной трубки перед горелкой и проплавляются ею, образуя прозрачный стеклообразный слой.

Процесс переноса оксидов легирующих веществ на внутреннюю поверхность трубы определяется действием термофоретических сил, возникающих ввиду наличия внутри трубки градиента температур. Опираясь на источники [1-6], в которых представлены результаты изучения процесса MCVD в России и за рубежом, можно выделить некоторые общие умозаключения, выполненные на базе фундаментальных исследованный, свойственные в общем случае процессам протекания физико-химических реакций в MCVD-процессе. 
1. Как следует из теории уравнений математической физики, температурному полю внутри кварцевой трубы, находящейся в зоне разогрева кислородо-водородной горелки, может быть поставлено в соответствие температурное поле поверхности кварцевой трубы, в частности профиль температурного распределения.

2. Эффективность образования оксидов в зоне окисления находится в жесткой зависимости от температурных условий. Причем для различных легирующих компонентов эти температуры различны, более того полнота протекания реакций окисления у каждого компонента своя. Эти знания позволяют обеспечивать требуемое температурное поле для оптимального с точки зрения полноты реакции процесса окисления паров легирующих компонентов.

3. Эффективность осаждения частиц под действием термофоретических сил определяется градиентом температур внутри трубы.

Движущаяся кислородо-водородная горелка - это подвижный тепловой источник воздействия, который создает определенное температурное поле в зоне разогрева кварцевой трубы. Температурное поле определяет эффективность протекания физико-химических реакций и качество заготовки ОВ.

В настоящее время активно развивается теория систем с распределенными параметрами, в том числе системы с подвижным источником воздействия [7-11]. Развитие технологий плавки, термообработки и напыления металлов с использованием электронно-лучевых и лазерных установок способствовало развитию систем управления с подвижным источником воздействия [12].

Объект управления в процессе MCVD характеризуется пространственной протяженностью, и его следует рассматривать как систему с распределенными параметрами.

Цель работы состоит в проектировании оптимального стабилизирующего регулятора температурного поля на поверхности кварцевой трубы в процессе MCVD.

\section{1. Описание объекта управления}

Будем считать, что нам заранее известно желаемое распределение температуры в цилиндрической трубе. Это известное распределение температуры и соответствующую ему интенсивность (мощность) теплового источника (при заданной скорости движения горелки) назовем 
оптимальным процессом. Оптимальное распределение температуры $\Theta^{*}(t, z)$ - оптимальная программная траектория - будет отличаться от фактического распределения температуры $\Theta(t, z)$ на величину $\Delta \Theta(t, z)$, причем величина отклонения $\Delta \Theta(t, z)$ будет небольшой относительно оптимальной программной траектории, т.е.

$$
\Theta(t, z)=\Theta^{*}(t, z)+\Delta \Theta(t, z)
$$

Аналогичным образом представим функцию управления $u(t, z)$ :

$$
u(t, z)=u^{*}(t, z)+\Delta u(t, z)
$$

где $u^{*}(t, z)$ - оптимальное программное управление; $u(t, z)$ - фактическое управление.

С учетом этого было получено линейное уравнение теплообмена для $\Delta \Theta(t, z)$, имеющее вид $[13,14]$

$$
\frac{\partial \Delta \Theta}{\partial t}-a \frac{\partial^{2} \Delta \Theta}{\partial z^{2}}+\alpha \frac{\partial \Delta \Theta}{\partial z}+\beta \Delta \Theta=\gamma^{2} \Delta u
$$

где $t$ - время; $z$ - пространственная переменная; $\Delta u(t, z)$ - управление; $\alpha, \beta$ и $\gamma-$ коэффициенты, полученные при линеаризации уравнения теплообмена.

Заметим, что в данной задаче управлением является мощность подвижного источника нагрева (горелки), тепловой поток $q(z, t)$ которого описывается функцией Гаусса [13]:

$$
q(z, t)=q_{\max } \cdot e^{-\left(\frac{z-\int_{0}^{t} v(\xi) d \xi}{H}\right)^{2}},
$$

где $v(\xi)$ - скорость движения теплового источника; $H$ - ширина пламени горелки; $q_{\max }-$ интенсивность (мощность) горелки.

Целью оптимального стабилизирующего управления является подбор такой интенсивности $q_{\max }$ горелки, при которой

$$
|\Delta \Theta(t, z)| \rightarrow 0
$$


В качестве функции управления $\Delta u(t, z)$ определим мощность горелки $q_{\text {max. }}$ Функционал для задачи оптимального управления (1) примет следующий вид [13]:

$$
F(\Delta u, \Delta \Theta)=\int_{0}^{\tau} \int_{0}^{L} \Delta \Theta^{2} d z d t+\sigma \int_{0}^{\tau} \int_{0}^{L} \Delta u^{2} d z d t,
$$

где $\sigma$ - параметр (цена управления).

Согласно работе [14] система оптимальности, соответствующая поставленной задаче, включает в себя дифференциальные уравнения для функции $\Delta \Theta(t, z)$ и сопряженной с ней вспомогательной функции $p(t, z)$ и имеет вид

$$
\left\{\begin{array}{l}
\frac{\partial \Delta \Theta}{\partial t}-a \frac{\partial^{2} \Delta \Theta}{\partial z^{2}}+\alpha \frac{\partial \Delta \Theta}{\partial z}+\beta \Delta \Theta=-\frac{\gamma^{2} p}{\sigma} \\
\frac{\partial p}{\partial t}+a \frac{\partial^{2} p}{\partial z^{2}}+\alpha \frac{\partial p}{\partial z}-\beta p=-\Delta \Theta \\
\left.\Delta \Theta\right|_{t=0}=\Theta_{0}(z),\left.p\right|_{t=\tau}=0 \\
\left.\Delta \Theta\right|_{z=0}=\Theta_{1}(z),\left.p\right|_{z=0}=0 \\
\left.\frac{\partial \Delta \Theta}{\partial z}\right|_{z=L}=\Theta_{2}(z),\left.\frac{\partial p}{\partial z}\right|_{z=L}=0
\end{array}\right.
$$

Важно отметить ключевую особенность системы оптимальности (7): она позволяет получить функции $\Delta \Theta(t, z)$ и $\Delta u(t, z)$ в аналитическом виде, которые могут быть использованы в режиме реального времени для систем автоматического управления.

Следует отдельно отметить, что управление скоростью движения горелки и расходом азота на обдув горелки в проектируемой системе управления осуществляется по программному закону.

\section{2. Проектирование оптимального стабилизирующего регулятора}

Поясним принцип действия проектируемой системы управления. Предположим, что длительность процесса MCVD равна $T$. Под процессом в данном случае будем иметь ввиду проход горелки в направлении подачи паро-газовой смеси от $z=0$ до $z=L$. Тогда для любого $t$ из 
$[0, T]$ нам известны распределение температуры $\Theta^{*}(t, z)$ и соответствующее управление $u^{*}(t, z)$. Пусть контроль фактической температуры осуществляется с помощью сканирующего пирометра через равные промежутки времени $\tau$, кратные $t$. Тогда решение задачи оптимального стабилизирующего управления тепловым источником будет происходить на временном промежутке $[0, \tau]$. Причем при $t_{1}=0\left(t_{1} \in[0, \tau]\right)$ нам известны значения

$$
\Delta \Theta(z, 0)=\Theta(z, 0)-\Theta^{*}(t, z),
$$

где $\Theta(z, 0)=\Theta(t, z)$ - это фактическая температура, полученная с помощью сканирующего пирометра в момент времени $t$. Принимая $\Delta \Theta(z, 0)$ за начальное условие, а граничные условия для $\Delta \Theta(t, z)-$ равными нулю, вычислим температуру $\Delta \Theta(t, z)$ и изменение во времени теплового потока (управления) $\Delta u(t, z)$. Это управление будет действовать только на временном промежутке $[0, \tau]$, по истечении которого происходит новый замер температуры, вычисляем $\Delta \Theta(z, 0)$ и т.д.

\section{3. Реализации алгоритма оптимального стабилизирующего управления}

Алгоритм оптимального стабилизирующего управления реализовывается на базе управляющего компьютера станка MCVD.

На рис. 2 представлена структурная схема основных модулей управляющего компьютера, задействованных в работе проектируемой системы управления.

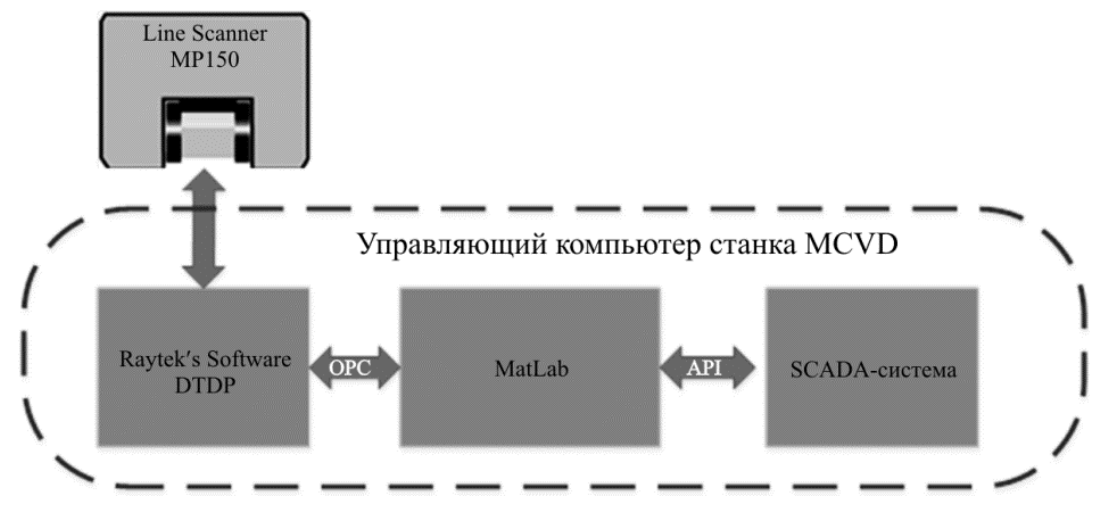

Рис. 2. Структурная схема управляющего компьютера 
Кратко опишем функции каждого модуля, изображенного на рис. 2.

Raytek's Software DTDP - специализированное программное обеспечение фирмы Raytek, позволяющее получать данные со сканирующего пирометра Raytek MP150. Обладает опцией OPC-сервера для передачи данных в другие приложения. В работе алгоритма, представленного на рис. 2, выступает в роли сервера фактического температурного поля объекта управления в каждый момент времени.

$M a t L a b$ - центральный модуль в реализации проектируемой системы управления. Алгоритм, изображенный на рис. 2, полностью реализуется на базе пакета MatLab. Ключевые задачи алгоритма: аппроксимация массива $\Delta \Theta(t, z)$ полиномиальной функцией и решение системы уравнений (7) - реализуются с помощью набора стандартных функций MatLab. Наличие собственного языка программирования позволяет реализовать логику работы алгоритма.

MatLab выступает в качестве OPC-клиента по отношению к Raytek's Software DTDP. MatLab обеспечивает двустороннюю связь co SCADA-системой станка MCVD, запрашивая величины фактических параметров процесса MCVD, участвующих в оптимальном стабилизирующем управлении, и передавая величину $\Delta u(t, z)$ для корректировки расхода водорода. Связь между пакетом MatLab и SCADAсистемой реализована через программный интерфейс приложения SCADA-системы (API).

$S C A D A$-система - программный пакет, предназначенный для разработки и обеспечения работы в реальном времени систем сбора, обработки, отображения и архивирования информации об объекте, а также возможного контроля и управления данным объектом. Двустороння связь между SCADA-системой MatLab позволяет осуществлять обмен ключевыми параметрами процесса, необходимыми для реализации алгоритма оптимального стабилизирующего управления: скоростью движения горелки, позицией горелки, расходом водорода на горелку, расходом азота на обдув горелки и др.

\section{Заключение}

В работе представлена система управления температурным полем, построенная на базе математической модели нагрева кварцевой трубы подвижным источником воздействия. Проектируемая система управления относится к классу систем с распределенными параметра- 
ми. Спроектирован оптимальный стабилизующий регулятор на основе представленной системы управления с распределенными параметрами. Реализован алгоритм оптимального стабилизирующего управления на базе математического пакета MatLab, адаптированный со SCADAсистемой станка MCVD.

\section{Список литературы}

1. Бурков В.Д., Иванов Г.А. Физико-технологические основы волоконно-оптической техники. - М.: ГОУ ВПО МГУЛ, 2007. - 222 с.

2. Choi M., Park K.S., Cho J. Modelling of chemical vapor deposition for optical fiber manufacture // Optical and Quantum Electronics. - 1995. № 27. - P. 327-335.

3. Dependence of equilibria in the modified chemical vapordeposition process on $\mathrm{SiCl}_{4}, \mathrm{GeCl}_{4}$, and $\mathrm{O}_{2} /$ K.B. Mcafee, K.L. Walker, R.A. Laudise, R.S. Hozack // Journal of the American Ceramic Society. 1984. - № 67 (6). - P. 420-424.

4. Nagel S.R., MacChesney J.B., Walker K.L. An overview of the modified chemical vapor deposition (MCVD) process and performance // IEEE Journal of Quantum Electronics. - 1982. - № 18 (4).

5. Cognolato L. Chemical vapour deposition for optical fiber technology // Journal de Physique IV. - 1995. - № 5.

6. Germanium chemistry in the MCVD process for optical fiber fabrication / D.L. Wood, K.L. Walker, J.B. Macchesney, J.R. Simpson, R. Csencsits // Journal of Lightwave Technology. - 1987. - № 5 (2).

7. Бутковский А.Г., Пустыльников Л.М. Теория подвижного управления системами с распределенными параметрами. - М.: Наука, 1980.

8. Кубышкин В.А. Подвижное управление колебаниями в системах с распределенными параметрами // Автомат. и телемех. - 2011. № 10. - С. 117-128.

9. Фурсиков А.В. Оптимальное управление распределенными системами. Теория и приложения: учеб. пособие. - Новосибирск: Научная книга, 1999. - 352 с.

10. Егоров А.И. Оптимальное управление тепловыми и диффузионными процессами. - М.: Наука, 1978. - 464 с.

11. Рапопорт Э.Я. Анализ и синтез систем автоматического управления с распределенными параметрами: учеб. пособие. - М.: Высшая школа, 2005. - 292 с. 
12. Кубышкин В.А., Суховеров В.С. Система моделирования и управления подвижным воздействием на базе программных средств MATLAB // Пробл. управл. - 2008. - № 2. - С. 64-69.

13. Первадчук В.П., Шумкова Д.Б. Оптимальное управление подвижным тепловым источником // Научно-технические ведомости СПбГПУ. - СПб., 2010. - С. 37-44.

14. Первадчук В.П., Владимирова Д.Б., Дектярев Д.Н. Оптимальное управление распределенными системами с подвижным тепловым источником // Наука и бизнес: пути развития. - 2016. - № 3 (57). C. 25-31.

\section{References}

1. Burkov V.D., Ivanov. G.A. Fiziko-tekhnologicheskiye osnovy volokonno-opticheskoy tekhniki. Moscow: GOU VPO MGUL. 2007, 222 p.

2. Choi M., Park K.S., Cho J. Modelling of Chemical Vapor Deposition for Optical Fiber Manufacture. Optical and Quantum Electronics, 1995, no. 27, pp. 327-335.

3. Mcafee K.B., Walker K.L., Laudise R.A., Hozack R.S. Dependence of equilibria in the modifed chemical vapor-deposition process on $\mathrm{SiCl}_{4}$. $\mathrm{GeCl}_{4}, \mathrm{O}_{2}$. Journal of the American Ceramic Society, 1984, no. 67 (6), pp. $420-424$.

4. Nagel S.R., MacChesney J.B., Walker K.L. An overview of the modifiedchemical vapor deposition (MCVD) process and performance. IEEE journal of quantum electronics, 1982, no. 18 (4).

5. Cognolato L. Chemical vapour deposition for optical fiber technology. Journal de physique IV. 1995, no. 5.

6. Wood D.L., Walker K.L., Macchesney J.B., Simpson J.R., Csencsits R. Germanium chemistry in the MCVD process for optical fiber fabrication. Journal of lightwave technology, 1987, no. 5 (2).

7. Butkovskiy A.G., Pustylnikov L.M. Teoriya podvizhnogo upravleniya sistemami s raspredelennymi parametrami. Moscow: Nauka, 1980.

8. Kubyshkin V.A. Podvizhnoye upravleniye kolebaniyami v sistemakh s raspredelennymi parametrami. Avtomatika i telemekhanika, 2011, no. 10, pp. 117-128.

9. Fursikov A.V. Optimalnoye upravleniye raspredelennymi sistemami. Teoriya i prilozheniya. Novosibirsk: Nauchnaya kniga, 1999, 352 p. 
10. Egorov A.I. Optimalnoye upravleniye teplovymi i diffuzionnymi protsessami. Moscow: Nauka, 1978, 464 p.

11. Rapoport E.Ya. Analiz i sintez sistem avtomaticheskogo upravleniya $\mathrm{s}$ raspredelennymi parametrami. Moscow: Vysshaya shkola, 2005, 292 p.

12. Kubyshkin V.A., Sukhoverov B.C. Sistema modelirovaniya i upravleniya podvizhnym vozdeystviyem na baze programmnykh sredstv MATLAB. Probl. upravl., 2008, no. 2, pp. 64-69.

13. Pervadchuk V.P., Shumkova D.B. Optimalnoye upravleniye podvizhnym teplovym istochnikom. Nauchno-tekhnicheskiye vedomosti SPbGPU, 2010, pp. 37-44.

14. Pervadchuk V.P., Vladimirova D.B., Dektyarev D.N. Optimalnoye upravleniye raspredelennymi sistemami $\mathrm{s}$ podvizhnym teplovym istochnikom. Nauka i biznes: puti razvitiya, 2016, no. 3 (57), pp. 25-31.

Получено 29.09.2017 